\title{
Ganho de peso na recria e desempenho reprodutivo de novilhas acasaladas com sobreano
}

\author{
Yuri Regis Montanholi(1), Júlio Otávio Jardim Barcellos ${ }^{(1)}$, João Batista Borges ${ }^{(2)}$, Eduardo Castro da Costa ${ }^{(1)}$, \\ Carolina Wunsh ${ }^{(1)}$ e Ênio Rosa Prates ${ }^{(1)}$
}

\begin{abstract}
(1)Universidade Federal do Rio Grande do Sul (UFRGS), Faculdade de Agronomia, Dep. de Zootecnia, Caixa Postal 776, CEP 91540-000 Porto Alegre, RS. E-mail: ymontanholi@yahoo.com, julio.barcellos@ufrgs.br (2)UFRGS, Faculdade de Medicina Veterinária, Dep. de Medicina Animal. E-mail: joao.borges@ufrgs.br
\end{abstract}

Resumo - O objetivo deste trabalho foi avaliar os efeitos da intensidade do ganho de peso no período de recria, dos 13 aos 18 meses de idade, sobre a taxa de prenhez de novilhas de corte acasaladas com o sobreano durante o outono. Os tratamentos foram constituídos de 30 novilhas com peso médio de $208 \mathrm{~kg}$, submetidas a ganho médio de $0,595 \mathrm{~kg} \mathrm{dia}^{-1}$ (G600), 41 novilhas com peso médio de $197 \mathrm{~kg}$ submetidas a ganho médio de $0,656 \mathrm{~kg} \mathrm{dia}^{-1}$ (G700) e 58 novilhas com peso médio de $181 \mathrm{~kg}$ submetidas a ganho médio de $0,723 \mathrm{~kg} \mathrm{dia}{ }^{-1}$ (G800). Foram avaliados os efeitos dos tratamentos sobre peso, escore de trato reprodutivo e taxa de prenhez. As taxas de prenhez foram 30,0\% 47,8\% e 50,0\% para os grupos G600, G700 e G800, respectivamente, não existindo diferença significativa entre os grupos. Os grupos G700 e G800, submetidos à maior taxa de ganho de peso, apresentaram maiores valores de escore de trato reprodutivo em relação ao G600 no início da estação de monta. Novilhas com escores mais elevados apresentaram tendência a maior taxa de prenhez. A taxa de prenhez esteve associada à intensidade de ganho de peso na recria independentemente das variações de peso no acasalamento.

Termos para indexação: bovinos de corte, escore de trato reprodutivo, rebanho de cria, taxa de prenhez, variação de peso.

\section{Live weight gain in the rearing and reproductive performance of yearling beef heifers mated}

\begin{abstract}
The objective of this work was to evaluate the effects of growth rate during rearing period, from 13 to 18 months, on pregnancy rate of beef heifers mated at yearling during autumn. Treatments consisted of 30 heifers weighing on average $208 \mathrm{~kg}$ submitted to average growth rate of $0.595 \mathrm{~kg} \mathrm{day}^{-1}$ (G600), 41 heifers averaging $197 \mathrm{~kg}$ with average growth rate of $0.656 \mathrm{~kg} \mathrm{day}^{-1}(\mathrm{G} 700)$ and 58 heifers with $181 \mathrm{~kg}$ with average growth rate of $0.723 \mathrm{~kg} \mathrm{day}^{-1}$ (G800). Treatment effects were determined on body weight, reproductive tract score and pregnancy rate. Pregnancy rate was $30.0 \% 47.8 \%$ and 50.0\%, respectively, for G600, G700 and G800, without significant differences among groups. Treatments G700 and G800, with large growth rates, showed higher values of reproductive tract score than G600 at the start of the mating period. Heifers with higher scores had a tendency to present higher pregnancy rates. Pregnancy rates were related to growth rate during postweaning independently of weight variations at mating.
\end{abstract}

Index terms: beef cattle, breeding herd, live weight change, pregnancy rate, reproductive tract scoring.

\section{Introdução}

Os sistemas de produção de bovinos de corte dependem do desempenho dos rebanhos de cria. Nestes, de $10 \%$ a $20 \%$ das vacas são substituídas anualmente por novilhas (Bolze \& Corah, 1993), para assegurar alta produtividade e eficiência do sistema. As novilhas de reposição são necessárias para manter estável o tamanho do rebanho e permitir o melhoramento ou alteração da sua base genética (Bagley, 1993).
A máxima eficiência biológica de um sistema de cria é alcançada quando as fêmeas são acasaladas entre 12 e 14 meses de idade (Short et al., 1994). Neste sistema existe o mínimo de categorias improdutivas (Fries, 2003). Entretanto, os custos associados a este sistema são os mais significativos, devido a sua alta tecnificação. Uma alternativa aos sistemas de produção que buscam maior produtividade por meio da redução na idade ao primeiro serviço é o acasalamento aos 18 meses, pois os custos envolvidos na produção de fêmeas para este sistema são 
reduzidos em relação ao sistema de 12 a 14 meses (Barcellos et al., 2003).

$\mathrm{O}$ acasalamento aos 18 meses determina que a novilha seja acasalada em uma estação do ano diferente do seu nascimento. Nas condições brasileiras de produção, normalmente os nascimentos se concentram na primavera, logo, o acasalamento aos 18 meses ocorre no outono. $\mathrm{O}$ aumento da utilização desta idade no primeiro serviço, como prática de manejo, irá determinar a existência de dois rebanhos distintos num mesmo sistema de cria, o de primavera e o de outono. Entretanto, devese considerar que o acasalamento com sobreano pode ser utilizado para assegurar um bom desempenho das vacas primíparas, mediante o primeiro intervalo de partos de 540 dias (Sampredo et al., 1995). Posteriormente, elas entram na estação reprodutiva da primavera, aos 36 meses, e concorrem para um intervalo de partos de 365 dias.

$\mathrm{O}$ acasalamento no outono permite que as fêmeas iniciem a estação de monta com melhor condição corporal em relação ao acasalamento na primavera, considerando o valor nutricional das pastagens nas estações que as antecedem, o verão e o inverno (Freitas et al., 1976). Deste modo, no outono as fêmeas, apresentam maiores chances de conceber no início da estação de monta. De acordo com Lesmeister et al. (1973), novilhas que concebem no início do período de acasalamento apresentam maior produtividade ao longo de suas vidas.

Novilhas de reposição, para serem incluídas no rebanho de cria, devem estar aptas a conceber. Para que estas fêmeas jovens apresentem adequado desempenho reprodutivo no primeiro acasalamento, devem apresentar atividade cíclica antes do início da estação de monta. De acordo com Byerley et al. (1987), novilhas acasaladas no terceiro ciclo estral apresentam melhor desempenho reprodutivo do que as novilhas acasaladas no estro pubertal. Desta maneira, no contexto de um sistema de produção, as novilhas devem estar púberes cerca de dois meses antes do início da estação de monta.

Entre os fatores que influenciam o surgimento da puberdade nos bovinos, destaca-se o peso vivo (Joubert, 1963). Associado a este fator, existem os efeitos da intensidade do ganho de peso (Wiltbank et al., 1969) e da fase pós-desmame, quando ocorre esse ganho (Dufour, 1975). Do ponto de vista biológico, deve-se salientar que a puberdade não é determinada pelo peso, mas por uma série de condições fisiológicas que resultam em um determinado peso.

Pesq. agropec. bras., Brasília, v.39, n.12, p.1253-1259, dez. 2004
Com o objetivo de proporcionar uma atenção especial ao trato reprodutivo das novilhas, Anderson et al. (1991) desenvolveram um sistema de escore do trato reprodutivo, que permite avaliar o grau de amadurecimento do aparelho genital das fêmeas de reposição. Este escore baseia-se na avaliação, por palpação retal, dos ovários e dos cornos uterinos. Diversos são os propósitos da utilização deste escore em sistema de cria, destacando-se sua aplicação no descarte de novilhas no processo de seleção para reposição e na definição ou adequação do programa nutricional das fêmeas antes do início da estação de monta.

O objetivo deste trabalho foi verificar a existência de relações entre a taxa de ganho de peso, durante o período da recria, e o desempenho reprodutivo de novilhas de corte acasaladas aos 18 meses no outono.

\section{Material e Métodos}

O experimento foi conduzido em propriedade particular, no Município de Bagé, região fisiográfica da Campanha do Estado do Rio Grande do Sul, situada entre os paralelos $30^{\circ} 30^{\prime}$ e $31^{\circ} 56^{\prime}$ latitude sul e os meridianos $55^{\circ} 30^{\prime}$ e $54^{\circ} 30^{\prime}$ longitude oeste de Greenwich. O período experimental foi compreendido entre 15/11/2002 e 20/9/2003. O clima da região, segundo a classificação de Köppen, é subtropical, da classe Cfa, com chuvas regularmente distribuídas durante o ano, podendo ocorrer períodos de estiagem nos meses de janeiro e fevereiro. A precipitação média anual é de $1.350 \mathrm{~mm}$, com variação de 1.080 a $1.620 \mathrm{~mm}$. A temperatura média anual é de $17,6^{\circ} \mathrm{C}$; a média do mês mais quente, janeiro, é $24^{\circ} \mathrm{C}$ e a do mês mais frio, junho, é $12,5^{\circ} \mathrm{C}$ (Moreno, 1961). Os solos da região são basicamente representados pelo tipo Cambissolo Háplico Ta eutrófico vértico (Embrapa, 1999), que apresentam variabilidade quanto à profundidade.

A pastagem nativa do local é formada por espécies de gramíneas rizomatosas e estoloníferas com a presença de leguminosas que crescem, preferencialmente, na primavera/verão. Destaca-se a presença de gramaforquilha (Paspalum notatum), campim-melador (Paspalum dilatatum), grama-tapete (Axonopus compressus), capim rabo-de-lagarto (Coelorachis selloana), capim-caninha (Andropogon lateralis), pega-pega (Desmodium pratensis) e trevo nativo (Tripholium polimorfum). Entre as espécies hibernais, destaca-se o capim-flexilha (Stipa spp.). 
No dia 15/11/2002, foram selecionados os animais para alocação em cada grupo experimental, de acordo com o peso médio. Os grupos foram denominados de G600 (30 novilhas), G700 (41 novilhas) e G800 (58 novilhas), cujos pesos foram 210, 195 e $175 \mathrm{~kg}$, respectivamente. Nesta ocasião os animais também foram identificados por meio de brincos numerados na orelha esquerda. Todos os animais pertenciam a raça Polled Hereford, apresentavam idade entre 13 e 14 meses e receberam, desde o desmame até o início do experimento, as mesmas condições de manejo.

Os grupos foram mantidos em lotações de $300 \mathrm{~kg} \mathrm{ha}^{-1}$ (G600), $200 \mathrm{~kg} \mathrm{ha}^{-1}$ (G700) e $150 \mathrm{~kg} \mathrm{ha}^{-1}$ (G800), que se constituíram nos sistemas de alimentação, com o objetivo de produzir distintos ganhos de peso nos animais de cada grupo, de maneira que todas as novilhas atingissem pesos similares, $300 \mathrm{~kg}$, no início do acasalamento (24/4/2003). Os animais foram pesados mensalmente e as cargas ajustadas conforme o ganho de peso no mês anterior, de forma a garantir um ganho de peso próximo do projetado. O ajuste da carga foi realizado com animais reguladores. A Tabela 1 apresenta os valores projetados e obtidos de peso inicial (PI), peso ao início do acasalamento (PIA) e de ganho diário médio (GDM).

Os piquetes utilizados eram bastante homogêneos quanto à topografia e composição botânica, com fácil acesso à água e suplementação mineral. As novilhas foram submetidas ao controle de endo e ectoparasitas e vacinadas contra as principais enfermidades da região. $\mathrm{O}$ experimento foi dividido em dois períodos, o de recria de 15/11/2002 a 23/4/2003 (160 dias), e o de acasalamento de 24/4/2003 a 7/6/2003 (45 dias).

Durante a recria foi realizada, mensalmente, a pesagem dos animais, no período da manhã mediante prévio jejum de 12 horas. No dia 23/4/2003, foi realizada a avaliação do escore do trato reprodutivo (ETR), por meio da palpação retal, conforme o método proposto por Anderson et al. (1991) (Tabela 2).

No período de acasalamento, de 24/4/2003 a 7/6/2003, foram realizadas duas pesagens, no período da manhã, mediante prévio jejum de 12 horas, nos dias 16/5/2003 e $7 / 6 / 2003$, com a finalidade de obter os pesos na metade e no final da estação de monta. Neste período, as novilhas foram mantidas em uma carga de $300 \mathrm{~kg} \mathrm{ha}^{-1}$. $\mathrm{O}$ acasalamento foi por meio de monta natural, utilizando-se um porcentual de $5 \%$ de touros, os quais foram

Tabela 1. Valores do peso inicial (PI), ganho diário médio (GDM) e peso ao início do acasalamento (PIA) projetados e obtidos, durante o período da recria, nos grupos experimentais de novilhas de corte.

\begin{tabular}{|c|c|c|c|c|c|c|}
\hline \multirow[t]{2}{*}{ Grupo $^{(1)}$} & \multicolumn{2}{|c|}{ PI (kg) } & \multicolumn{2}{|c|}{ GDM $\left(\mathrm{kg} \mathrm{dia}^{-1}\right)$} & \multicolumn{2}{|c|}{ PIA (kg) } \\
\hline & Projetado & Observado & Projetado & Observado & Projetado & Observado \\
\hline G600 & 210 & 208 & 0,563 & 0,595 & 300 & 302 \\
\hline G700 & 195 & 197 & 0,656 & 0,637 & 300 & 298 \\
\hline G800 & 175 & 181 & 0,781 & 0,723 & 300 & 296 \\
\hline
\end{tabular}

${ }^{(1)}$ G600: 30 novilhas mantidas na lotação de $300 \mathrm{~kg} \mathrm{ha}^{-1}$; G700: 41 novilhas mantidas na lotação de 200 kg ha-1; G800: 58 novilhas mantidas na lotação de $150 \mathrm{~kg} \mathrm{ha}^{-1}$.

Tabela 2. Descrição do escore de trato reprodutivo (ETR) em novilhas de corte.

\begin{tabular}{|c|c|c|c|c|c|}
\hline \multirow[t]{3}{*}{ ETR } & \multirow[t]{3}{*}{ Cornos uterinos } & \multicolumn{4}{|c|}{ Ovário } \\
\hline & & Comprimento & Altura & Largura & Folículos \\
\hline & & \multicolumn{4}{|c|}{ 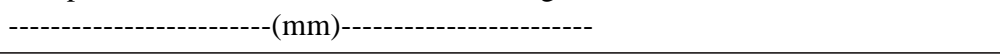 } \\
\hline 1 & $\begin{array}{l}\text { Imaturo }<20 \mathrm{~mm} \text { de } \\
\text { diâmetro, sem tônus }\end{array}$ & 15 & 10 & 8 & Ausência \\
\hline 2 & $\begin{array}{l}20-25 \mathrm{~mm} \text { de diâmetro, } \\
\text { sem tônus }\end{array}$ & 18 & 12 & 10 & $8 \mathrm{~mm}$ \\
\hline 3 & $\begin{array}{l}25-30 \mathrm{~mm} \text { de diâmetro, } \\
\text { leve tônus }\end{array}$ & 22 & 15 & 10 & $8-10 \mathrm{~mm}$ \\
\hline 4 & $\begin{array}{l}30 \mathrm{~mm} \text { de diâmetro, } \\
\text { bom tônus }\end{array}$ & 30 & 16 & 12 & $>10 \mathrm{~mm}$ \\
\hline 5 & $\begin{array}{l}>30 \mathrm{~mm} \text { de diâmetro, } \\
\text { bom tônus, ereto }\end{array}$ & $>32$ & 20 & 15 & $>10 \mathrm{~mm}$, com corpo lúteo \\
\hline
\end{tabular}

Fonte: Anderson et al. (1991). 
previamente selecionados mediante exame andrológico. O diagnóstico de gestação, pela palpação retal foi realizado, em 20/9/2003, 104 dias após o fim da estação de monta.

As estimativas da disponibilidade de matéria seca (MS) por hectare foram realizadas, mensalmente, por meio de quatro cortes rente ao solo com um quadrado de $0,25 \mathrm{~m}^{2}$. Foi realizada a separação das amostras em material senescido e material verde. Também foram realizadas coletas de amostras nos locais que haviam sido pastejados recentemente, para se obter maior precisão do alimento consumido pelos animais.

As amostras coletadas da pastagem nativa foram processadas no Laboratório de Nutrição Animal da Universidade Federal do Rio Grande do Sul. Foram realizadas as análises de proteína bruta (PB), pelo método Kjeldahl, fibra detergente neutro (FDN) conforme o método de Van Soest \& Robertson (1985) e dos teores de MS e cinzas (CZ). Foram avaliados os pesos, os GDM durante a recria, a variação diária média de peso durante o acasalamento (VDA), os valores do ETR e a taxa de prenhez (TP).

A TP foi determinada com base nos resultados do diagnóstico de gestação e foi analisada pelo teste do qui-quadrado. A análise das variáveis de resposta contínua foi realizada por meio da análise da variância. $\mathrm{O}$ efeito do ganho de peso médio sobre a TP e o ETR foram determinados por meio da análise de regressão simples. As diferenças entre as médias ajustadas foram testadas pelo teste de Tukey, segundo o modelo:

$\mathrm{Yij}=\mu+\mathrm{Gi}+\varepsilon \mathrm{ij}$,

em que Yij é a j-ésima resposta medida no i-ésimo grupo de novilhas; $\mu$ é o efeito médio; Gi é o efeito do ganho de peso; $\varepsilon i j$ é o j-ésimo erro associado à i-ésima resposta.

\section{Resultados e Discussão}

Opeso no início do acasalamento (PIA) estabelecido pelo desenho experimental representa cerca de $65 \%$ do peso adulto (460 kg) das vacas do rebanho utilizado (Tabela 3). De acordo com Bolze \& Corah (1993), um PIA equivalente a $65 \%$ do peso da vaca adulta pode assegurar elevadas taxas de prenhez (TP) no primeiro acasalamento. Embora o PIA seja uma variável fundamental na determinação da prenhez, neste experimento o objeto de avaliação foi a taxa de ganho de peso prévia ao acasalamento das novilhas que alcançaram um mesmo peso alvo.

As novilhas do G600 e do G700 apresentaram pequenos aumentos nos valores do peso mensurado durante o período de acasalamento (PMA) em relação ao PIA (Tabela 3). Por outro lado, houve queda no peso das novilhas do G800, que vinham de uma carga de $150 \mathrm{~kg} \mathrm{ha}^{-1}$ e no início do acasalamento foram submetidas, com o G600 e G700, a uma carga de $300 \mathrm{~kg} \mathrm{ha}^{-1}$, o que pode ter influenciado os resultados deste grupo, determinando a diminuição no seu peso vivo. Com relação à variação de peso durante todo o período de acasalamento é provável que, com o avançar do outono, quando as espécies estivais já se encontram em processo de senescência, diminuindo o valor nutricional da pastagem nativa (Freitas et al., 1976), o ganho de peso dos três grupos tenha sido prejudicado. Isto fica evidente quando se observa a variação de peso na segunda metade do período de acasalamento, sendo observadas perdas superiores a $0,220 \mathrm{~kg} \mathrm{dia}^{-1}$ quanto aos três grupos.

A variação diária de peso durante o acasalamento (VDA) diverge do resultado obtido por Silva (2003), que trabalhou em condições similares no mesmo local e observou uma VDA de $0,728 \mathrm{~kg} \mathrm{dia}^{-1}$. A magnitude desta diferença nos resultados de VDA pode ser atribuída a estrutura da pastagem, uma vez que os dois experimentos foram conduzidos com uma mesma carga animal (300 $\left.\mathrm{kg} \mathrm{ha}^{-1}\right)$, porém com diferentes disponibilidades. De acordo com Correa \& Maraschin (1994), o nível de oferta de pastagem nativa apresenta um comportamen-

Tabela 3. Peso no início do acasalamento (PIA), peso obtido durante a estação de monta (PMA), peso no fim do acasalamento (PFA), variação diária no peso durante o acasalamento (VDA), escore de trato reprodutivo (ETR) e taxa de prenhez (TP), em relação aos grupos experimentais de novilhas de corte ${ }^{(1)}$.

\begin{tabular}{lcccccc}
\hline Grupo $^{(2)}$ & PIA $(\mathrm{kg})$ & PMA $(\mathrm{kg})$ & PFA $(\mathrm{kg})$ & VDA $\left(\mathrm{kg} \mathrm{dia}^{-1}\right)$ & ETR & TP $(\%)$ \\
\hline G600 & $302,8 \mathrm{a}$ & $304,4 \mathrm{a}$ & $298,1 \mathrm{a}$ & $-0,103 \mathrm{a}$ & $3,00 \mathrm{a}$ & $30,0 \mathrm{a}$ \\
G700 & $298,0 \mathrm{~b}$ & $299,3 \mathrm{a}$ & $293,1 \mathrm{ab}$ & $-0,108 \mathrm{a}$ & $3,35 \mathrm{ab}$ & $47,8 \mathrm{a}$ \\
G800 & $296,5 \mathrm{~b}$ & $291,1 \mathrm{~b}$ & $286,3 \mathrm{~b}$ & $-0,228 \mathrm{~b}$ & $3,96 \mathrm{~b}$ & $50,0 \mathrm{a}$ \\
\hline
\end{tabular}

${ }^{(1)}$ Médias seguidas por letras diferentes na coluna diferem entre si pelo teste de Tukey $(\mathrm{P}<0,06)$, exceto em TP, analisada pelo teste de qui-quadrado $(\mathrm{P}<0,05) .{ }^{(2)} \mathrm{G} 600$ : 30 novilhas mantidas na lotação de $300 \mathrm{~kg} \mathrm{ha}^{-1} ; \mathrm{G} 700: 41$ novilhas mantidas na lotação de $200 \mathrm{~kg}$ ha-1; G800: 58 novilhas mantidas na lotação de $150 \mathrm{~kg} \mathrm{ha}^{-1}$. 
to quadrático sobre o ganho de peso dos bovinos. As condições climáticas também podem ter afetado a VDA. Durante a estação de monta, foram observados 11 dias com precipitações pluviais superiores a $10 \mathrm{~mm}$, o que representa cerca de $25 \%$ do período da estação de monta. Segundo o National Research Council (2000), a nutrição e o estresse ambiental são inter-relacionados podendo ser afetados diretamente pelas condições climáticas, como a precipitação pluvial.

A TP não foi diferente entre os grupos experimentais ( $\mathrm{P}>0,05)$, o que pode ser atribuído ao reduzido número de animais utilizados (Tabela 3). Contudo, foi observada tendência de maior TP nos grupos G700 e G800 em relação ao G600. Isto sugere que, embora estes grupos tenham apresentado menor $(\mathrm{P}<0,06)$ PIA, a maior taxa de ganho de peso durante a recria parece ter proporcionado maior desenvolvimento do aparelho reprodutivo destas novilhas, conforme observado nos valores de ETR, resultando em maior fertilidade. A análise de regressão entre ganho diário médio (GDM) e TP demonstra tendência de comportamento linear $(\mathrm{P}<0,10)$ entre estes dois parâmetros (Figura 1). À medida que se observa maior GDM na recria, maiores TP para as novilhas são esperadas.

Em novilhas agrupadas de acordo com os valores de ETR (Tabela 4) foi evidenciada a associação entre o ETR e a TP, demonstrando a validade da ferramenta para estimar o desempenho reprodutivo dos grupos de animais. Embora a diferença na TP entre os grupos não tenha sido identificada $(\mathrm{P}>0,05)$, observa-se tendência de maior TP conforme maior valor para ETR. Esta evidência está de acordo com Rosenkrans \& Hardin (2003), que recomendam a utilização do ETR em grupos de animais, e não para avaliar os animais individualmente.

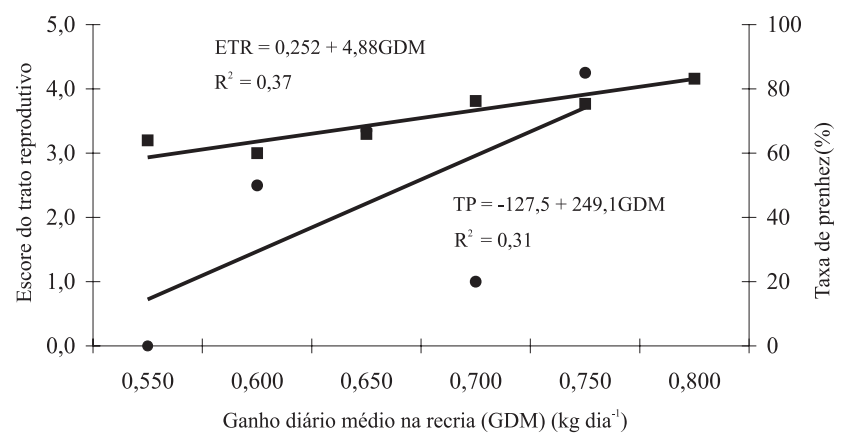

Figura 1. Regressão do ganho diário médio na recria sobre o escore de trato reprodutivo $(\bullet$, ETR) e taxa de prenhez $(\bullet$, TP) de novilhas de corte.
As TP observadas em categorias de ETR consideradas são inferiores aos resultados apresentados por Anderson et al. (1991). Isto pode ser atribuído aos prejuízos causados pela VDA no desempenho reprodutivo das novilhas. Segundo Anderson et al. (1991) novilhas com ETR igual a 3 apresentam condições de se tornarem púberes e conceberem durante a estação de monta. Entretanto, a função reprodutiva é limitada em condições de restrição nutricional, como a observada neste trabalho. De acordo com Day et al. (1986), a restrição do nível energético deprime o eixo hipófise-hipotalâmico, o que diminui a atividade ovariana. Desta maneira, supõe-se que até mesmo novilhas púberes diminuíram a atividade cíclica, o que proporcionou baixa TP para estas fêmeas. Esta argumentação é reforçada mediante a observação dos resultados de Ferreira et al. (1999), que observaram 56,2 e $84,5 \%$ de atividade cíclica em novilhas que apresentavam ETR 2 e 3, respectivamente, e que foram submetidas a moderados ganhos de peso durante $o$ acasalamento. A análise de regressão entre $o$ GDM e o ETR demonstra comportamento linear $(\mathrm{P}<0,05)$ entre estes dois parâmetros (Figura 1). À medida que se observa maior GDM na recria, espera-se maior desenvolvimento do aparelho reprodutivo.

O grande porcentual de dias com precipitações pluviométricas superiores a $10 \mathrm{~mm}$ durante a estação de monta também pode ter prejudicado a manifestação etológica da atividade sexual dos animais. De acordo com Hafez (1975), a manifestação sexual é fortemente reduzida em períodos de estresse fisiológico causado, entre outros fatores, pela precipitação.

Em novilhas agrupadas de acordo com a variação do peso no acasalamento, nas categorias I, II e III (Tabela 5), não foram verificadas diferenças $(\mathrm{P}>0,05)$ para ETR e PIA, evidenciando que a perda de peso durante o acasalamento não foi associada a estes parâmetros. Por sua vez, foram verificados maiores ganhos $(\mathrm{P}<0,05)$ de peso nos 60 dias anteriores à estação de monta (GD60) nas categorias II e III, as quais apresentaram maiores perdas de peso na estação de monta. De acordo

Tabela 4. Escore de trato reprodutivo (ETR) e taxa de prenhez (TP), em novilhas de corte agrupadas de acordo com o $\operatorname{ETR}^{(1)}$.

\begin{tabular}{lccc}
\hline Parâmetro & $\begin{array}{c}\text { Púbere } \\
(\mathrm{ETR}>3)\end{array}$ & $\begin{array}{c}\text { Pré-púbere } \\
(\mathrm{ETR}=3)\end{array}$ & $\begin{array}{c}\text { Infantil } \\
(\mathrm{ETR}<3)\end{array}$ \\
\hline ETR & $4,38 \mathrm{a}$ & $3,00 \mathrm{~b}$ & $1,92 \mathrm{c}$ \\
TP $(\%)$ & $50,0 \mathrm{a}$ & $40,9 \mathrm{a}$ & $30,8 \mathrm{a}$ \\
\hline
\end{tabular}

${ }^{(1)}$ Médias seguidas por letras diferentes na linha diferem entre si $(\mathrm{P}<0,05)$ pelo teste de Tukey (ETR) e pelo teste de qui-quadrado (TP). 
Tabela 5. Escore de trato reprodutivo (ETR), peso no início do acasalamento (PIA), ganho diário médio dos 60 dias anteriores ao início da estação de monta (GD60), variação do peso no acasalamento (VPA) e taxa de prenhez (TP), de acordo com as categorias agrupadas pela VPA em novilhas de corte ${ }^{(1)}$.

\begin{tabular}{lccccc}
\hline Variação de peso $\left(\mathrm{kg} \mathrm{dia}^{-1}\right)$ & ETR & PIA (kg) & GD60 $\left(\mathrm{kg} \mathrm{dia}^{-1}\right)$ & VPA $\left(\mathrm{kg} \mathrm{dia}^{-1}\right)$ & TP $(\%)$ \\
\hline I $(<-0,100)$ & $3,42 \mathrm{a}$ & $300,9 \mathrm{a}$ & $0,692 \mathrm{a}$ & $0,036 \mathrm{a}$ & $41,7 \mathrm{a}$ \\
II $(-0,100 \mathrm{a}-0,210)$ & $3,22 \mathrm{a}$ & $297,2 \mathrm{a}$ & $0,805 \mathrm{~b}$ & $-0,157 \mathrm{~b}$ & $33,3 \mathrm{a}$ \\
III $(>-0,210)$ & $3,64 \mathrm{a}$ & $298,2 \mathrm{a}$ & $0,816 \mathrm{~b}$ & $-0,322 \mathrm{c}$ & $52,0 \mathrm{a}$ \\
\hline
\end{tabular}

${ }^{(1)}$ Médias seguidas por letras diferentes na coluna diferem entre si pelo teste de Tukey (ETR, PIA, GD60 e VPA) $(\mathrm{P}<0,05)$ e pelo teste de quiquadrado $(\mathrm{P}<0,05)(\mathrm{TP})$.

com Freetly et al. (2000), um período de alto nível nutricional seguido por um de restrição alimentar proporciona maiores perdas de peso nos animais mantidos em níveis nutricionais, prévios a restrição, mais elevados.

Não foram identificadas diferenças $(\mathrm{P}>0,05)$ de TP nas categorias consideradas (Tabela 5), embora exista uma diferença numérica de 18,7 pontos porcentuais entre a TP das categorias II e III. Sawyer et al. (1991) observaram que novilhas com VPA nas faixas de $-0,600$ a $-0,100$, de $-0,100$ a 0,100 e 0,100 a $0,600 \mathrm{~kg} \mathrm{dia}^{-1} \mathrm{du}-$ rante a estação de monta, apresentaram $43 \%, 55 \%$ e $70 \%$ de TP, respectivamente. Esta relação apresentada por Sawyer et al. (1991) também é evidenciada em fêmeas adultas (Wiltbank et al., 1969). A tendência de maior TP em fêmeas da categoria III (Tabela 5) se deve ao maior GD60 destas, que provavelmente proporcionou um efeito de flushing, permitindo maior desenvolvimento das estruturas ovarianas (Mourasse et al., 1985), conforme se observa à tendência de maior ETR $(\mathrm{P}>0,05)$ nas fêmeas da categoria III, mas que vinham de maiores GD60. Este efeito parece não ter ocorrido nas novilhas da categoria II que embora, tenham obtido um GD60 similar ao das novilhas da categoria III, apresentaram tendência $(\mathrm{P}>0,05)$ de menor ETR em relação às demais categorias, o que repercutiu em uma tendência de menor TP para estas fêmeas.

A tendência $(\mathrm{P}>0,05)$ de maior TP para a categoria I em relação à categoria II, pode ser explicada pela VPA. $\mathrm{O}$ fato de novilhas da categoria I terem apresentado um pequeno ganho de peso no acasalamento e a tendência $(\mathrm{P}>0,05)$ de maior ETR no início da estação de monta para estas fêmeas, provavelmente, garantiu maior porcentual de fêmeas ciclando ou que iniciaram a atividade cíclica durante a estação de monta. Isto é melhor evidenciado quando se observa o ganho de peso de $0,143 \mathrm{~kg} \mathrm{dia}^{-1}$ na primeira metade do período de acasalamento em fêmeas da categoria I. Por sua vez, as novilhas da categoria II apresentavam, inicialmente, tendência de menor $(\mathrm{P}>0,05)$ ETR associada à perda de, aproximadamente, $0,157 \mathrm{~kg} \mathrm{dia}^{-1}$. Isto, provavelmente, prejudicou o desenvolvimento do aparelho reprodutivo destas fêmeas.

Diante dos resultados obtidos neste trabalho e as evidências de outros autores, sugere-se que maiores taxas de prenhez, mantendo as vantagens de elevadas taxas de ganho na recria, podem ser alcançadas por meio de melhores condições ambientais ou nutricionais durante a estação de monta. Um dos caminhos para se delinear este cenário pode ser o deslocamento do período de monta de outono para o início ou final da estação. Contudo, seus efeitos ainda não foram completamente elucidados.

\section{Conclusões}

1. Novilhas recriadas em taxas de ganho mais altas apresentam maiores valores de escore de trato reprodutivo, resultante do mais rápido amadurecimento do sistema reprodutivo.

2. A intensidade da perda de peso no acasalamento é associada ao ganho de peso nos 60 dias anteriores ao início da estação de monta.

3. A taxa de ganho de peso na recria é mais importante do que a variação de peso durante o acasalamento na determinação da taxa de prenhez em novilhas de corte acasaladas aos 18 meses de idade no outono.

\section{Referências}

ANDERSON, K.J.; LEFEVER, D.G.; BRINKS, J.S.; ODDE, K.G. The use of reproductive tract scoring in beef heifers. Agri-Practice, v.12, p.19-26, 1991.

BAGLEY, C.P. Nutritional management of replacement beef heifers: a review. Journal of Animal Science, v.71, p.3155-3163, 1993.

BARCELlOS, J.O.J.; COSTA, E.C.; SILVA, M.D.; SEMMELMANN, C.E.N.; MONTANHOLI, Y.R.; PRATES, E.R.; MENDES, R.; WUNSCH, C.; ROSA, J.R.P. Crescimento de fêmeas bovinas de corte aplicado aos sistemas de cria. Porto 
Alegre: UFRGS, 2003. 72p. (Sistemas de Produção em Bovinos de Corte. Publicação Ocasional, 1).

BOLZE, R.; CORAH, L.R. Selection and development of replacement heifers. Manhattan: Kansas State University, Cooperative Extension Service, 1993. 10p.

BYERLEY, D.J.; STAIGMILLER, R.B.; BERARDINELLI, J.G.; SHORT, R.E. Pregnancy rates of beef heifers bred either on puberal or third estrus. Journal of Animal Science, v.65, p.645-650, 1987.

CORREA, F.L.; MARASCHIN, G.E. Crescimento e desaparecimento de uma pastagem nativa sob diferentes níveis de oferta de forragem. Pesquisa Agropecuária Brasileira, v.29, p.1617-1623, 1994.

DAY, M.L.; IMAKAWA, K.; ZALESKY, D.D.; KITTOK, R.J.; KINDER, J.E. Effects of restriction of dietary energy intake during the prepubertal period on secretion of luteinizing hormone and responsiveness of the pituitary to luteinizing hormone-releasing hormone in heifers. Journal of Animal Science, v.62, p.1641-1648, 1986.

DUFOUR, J.J. Influence of postweaning growth rate on puberty and ovarian activity in heifers. Canadian Journal of Animal Science, v.55, p.93-100, 1975.

EMBRAPA. Centro Nacional de Pesquisa de Solos (Rio de Janeiro, RJ). Sistema brasileiro de classificação de solos. Brasília: Embrapa-SPI; Embrapa-CNPS, 1999. 412p.

FERREIRA, M.B.D.; LOPES, B.C.; DANTAS, M.S.; MOURÃO, G.B.; VALE FILHO, V.R. Escore do aparelho reprodutivo pré estação de monta em novilhas zebu aos dois anos de idade. Revista Brasileira de Reprodução Animal, v.23, p.160-162, 1999.

FREETLY, H.C.; FERREL, C.L.; JENKINS, T.G. Timing of realimentation of mature cows that were feed-restricted during pregnancy influences calf birth weights and growth rates. Journal of Animal Science, v.78, p.2790-2796, 2000.

FREITAS, E.A.G.; LÓPEZ, J.; PRATES, E.R. Produtividade, matéria seca, proteína digestível e nutrientes digestíveis totais em pastagem nativa do Rio Grande do Sul. Anuário Técnico do IPZFO, v.3, p.454-515, 1976.

FRIES, L.A. Genética para um sistema de produção de ciclo curto. In: SIMPÓSIO DA CARNE BOVINA, 1., 2003, São Borja. Anais. Porto Alegre: Ed. UFRGS, 2003. p.47-82.

HAFEZ, E.S.E. (Ed.). The behaviour of domestic animals. London: Baillière Tindall, 1975. 532p.
JOUBERT, D.M. Puberty in female farm animals. Animal Breeding Abstracts, v.31, p.295-306, 1963.

LESMEISTER, J.L.; BURFENING, P.J.; BLACKWELL, R.L. Date of first calving in beef cows and subsequent calf production. Journal of Animal Science, v.36, p.1-6, 1973.

MOREnO, J.A. Clima do Rio Grande do Sul. Porto Alegre: Secretaria da Agricultura, 1961. 42p.

MOURASSE, C.; MATTON, P.; DUFOUR, J.J. Ovarian follicular populations at two stages of an estrous cycle in heifers given high energy diets. Journal of Animal Science, v.61, p.1194-1200, 1985.

NATIONAL RESEARCH COUNCIL. Nutrient requirements of beef cattle. $7^{\text {th }}$ ed. rev. Washington, DC: National Academy of Sciences, 2000. 234p.

ROSENKRANS, K.S.; HARDIN, D.K. Repeatability and accuracy of reproductive tract scoring to determine pubertal status in beef heifers. Theriogenology, v.59, p.1087-1092, 2003.

SAMPEDRO, D.; VOGEL, O.; CELSER, R. Alternativas de manejo para entorar la vaquilla a los 18 meses de edad: su influencia sobre el porcentaje de 20 entore y prenhez. Mercedes: INTA, 1995. 9p. (Circular Técnica).

SAWYER, G.J.; BARKER, D.J.; MORRIS, R.J. Performance of young breeding cattle in comercial herds in the south-west of Western-Australia: 1. Liveweight, body condition, conception and fertility in heifers. Australian Journal of Experimental Agriculture, v.31, p.431-441, 1991.

SHORT, R.E.; STAIGMILLER, R.B.; BELLOWS, R.A.; GREER, R.C. Breeding heifers at one year of age: biological and economic considerations. In: FIELDS, M.J.; SAND, R.S. (Ed.). Factors affecting calf crop. Boca Raton: CRC Press, 1994. p.55-68.

SILVA, M.D. Desempenho reprodutivo de novilhas de corte acasaladas aos 18 ou 24 meses de idade. 2003. 107p. Dissertação (Mestrado) - Universidade Federal do Rio Grande do Sul, Porto Alegre.

VAN SOEST, P.J.; ROBERTSON, J.B. Analysis of forages and fibrous foods: a laboratory manual for Animal Science. Ithaca: Cornell University Press, 1985. 202p.

WILTBANK, J.N.; KASSON, C.W.; INGALLS, J.E. Puberty in crossbred and straightbred beef heifers on two levels of feed. Journal of Animal Science, v.29, p.602-605, 1969. 